IAU Colloquium 164: Radio Emission from Galactic and Extragalactic Compact Sources

ASP Conference Series, Vol. 144,1998

J. A. Zensus, G. B. Taylor, \& J. M. Wrobel (eds.)

\title{
VLBI Observations of Gravitational Lenses
}

\author{
R. W. Porcas \\ Max-Planck-Institut für Radioastronomie, Bonn, Germany
}

Abstract. The application of the VLBI technique to the study of gravitational lenses is described, and results from recent VLBI observations are reviewed.

\section{Lens Systems: Searching, Confirming and Imaging}

VLBI can be used to study the images of gravitational lens systems, providing the background object is a sufficiently strong and compact radio source. To date there are 9 published sources which are identified as multiply-imaged lens systems with reasonable certainty. Four are 2 -image systems $(0218+357$, $0957+561,1600+434,1830-211)$ and five are 4-image "quads" (MG 0414+0534, $1422+231,1608+656,1938+666,2016+112)$. VLBI observations of 7 of these systems, published up to 1995, are reviewed in Patnaik \& Porcas (1996).

From the discovery of the first lens, 0957+561, 18 years ago, VLBI has been used to study the morphology and similarity of compact image components, as a method of confirming lens candidates (Porcas et al. 1979). VLBI observations of several new candidates are currently underway (e.g., 0712+472, 1030+074, $2114+022$; Wilkinson \& Browne, private communication) in an effort to increase the number of known lenses.

The unique resolution provided by VLBI can also be used to search for "milli-lenses" (with image separations from 1-100 mas), which would result from compact lenses with masses of $10^{6}-10^{8} \mathrm{M}_{\odot}$ (e.g., Patnaik et al. 1996; Wilkinson et al. 1996). Although there are no confirmed cases yet, such studies can constrain the amount of dark matter in the Universe in the form of compact objects in this mass range.

The high resolution of VLBI also results in special imaging problems, particularly for weak sources, because of the wide field-of-view necessary to include all the images. A number of special techniques have been developed to overcome these (e.g., Porcas 1994; Garrett et al. 1994b)

\section{Recent Observations of Confirmed Lens Systems}

Detailed VLBI studies of the images of confirmed systems can help clarify the lensing process and put constraints on the mass distribution of the lens. Although the double and quad image configurations can easily be modeled by single galaxy lenses with elliptical (projected) mass distributions, the "quads" $1938+666$ (King et al. 1997) and 2016+112 (Garrett et al. 1996a) seem to require complex background sources or more complicated lenses. $2016+112$ consists of 2 similar image components, $\mathrm{A}$ and $\mathrm{B}$, and a complex, elongated region $\mathrm{C}$, which EVN observations have shown to consist of at least 4 sub-components located on a gentle arc (see Fig. 1). Recent EVN observations at $5 \mathrm{GHz}$ reveal similar, double structures in $\mathrm{A}$ and $\mathrm{B}$, and show that the central two sub-components in $\mathrm{C}$ resemble the "merging images", which straddle a critical line in the image 

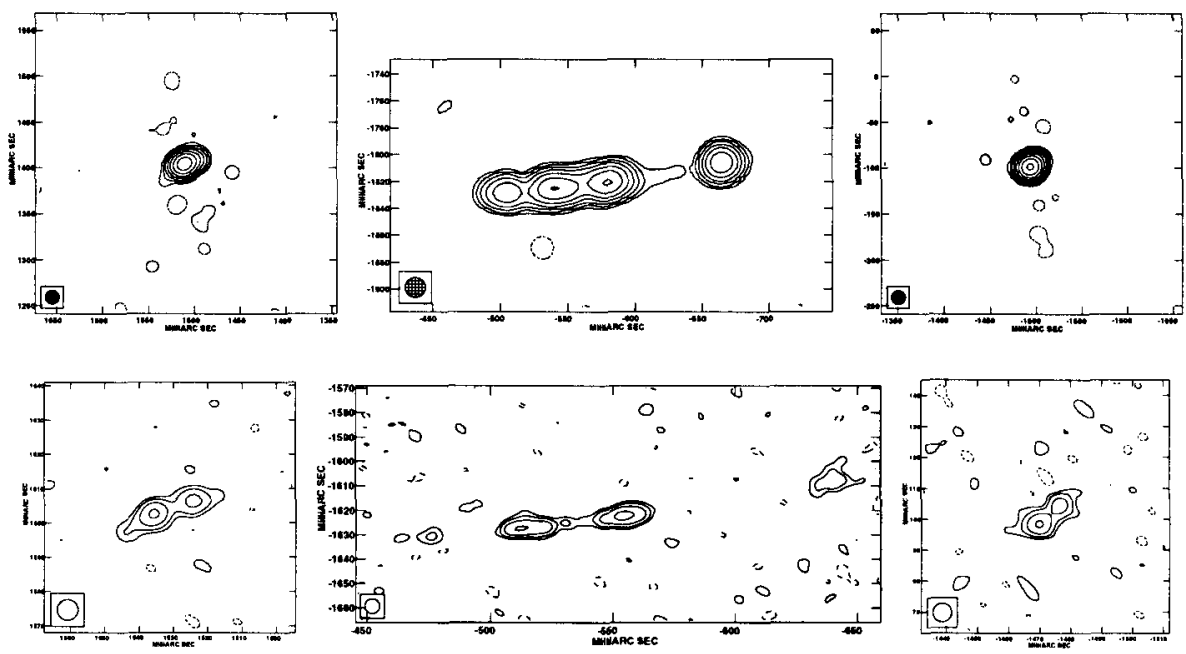

Figure 1. EVN observations of $2016+112$, images (l to r) A,C,B; Top: $1.6 \mathrm{GHz}$ maps (from Garrett et al. 1996a); Bottom: $5 \mathrm{GHz}$ maps (from Garrett, Porcas, \& Patnaik, in preparation)

when the background source crosses a caustic. Nair \& Garrett (1997) have produced two models which both incorporate two galaxies at different redshifts; in one model, some of the subcomponents in $\mathrm{C}$ originate from a different source from that producing images $\mathrm{A}$ and $\mathrm{B}$.

The double 1830-211 comprises 2 compact image components (NE,SW) surrounded by an Einstein ring of emission. Low resolution VLBI observations reveal similar core-jet structures in the images (of opposite parity, as expected), the jets pointing to the SE and NW respectively. Higher resolution observations, however, reveal a NW pointing jet in the NE image (Jones et al. 1996; Garrett et al. 1997) which has no apparent counterpart in the SW image (Figure 2). Garrett et al. (these Proceedings, p. 313) have also observed dramatic changes in the inner cores of both images, monitored at $43 \mathrm{GHz}$ using the VLBA. The presence of absorption lines at 2 different redshifts in $1830-211(z=0.89,0.19)$ has led Lovell et al. (1996) to suggest that 2 galaxies combine to form a compound lens in this system. From a study of the increase of image size with wavelength, Jones et al. have also suggested that image broadening occurs due to interstellar scattering in the Galaxy, as the image paths pass close to the Galactic center (Figure 3).

Wambsganss \& Paczynski (1992) have suggested that detailed studies of the mas structures of lensed images can reveal the presence of mass inhomogeneities in the lens. Garrett et al. (1996b) have listed some of the potential problems which arise when attempting this in practice, as in the case of 1830211. However, the long, 50 mas jets of the $\mathrm{A}$ and $\mathrm{B}$ images of $0957+561$ have been examined using $1.6 \mathrm{GHz}$ global VLBI (Garrett et al. 1994a). The absence of any large distortions in one image, not seen in the other, puts a limit of $<10$ 
$\%$ for the amount of dark matter in the halo of the lens galaxy in the form of $>3 \cdot 10^{6} \mathrm{M}_{\odot}$ black holes.

One of the "holy grails" of gravitational lens studies is to derive a direct estimate of the Hubble constant by making a detailed mass model of the lens,
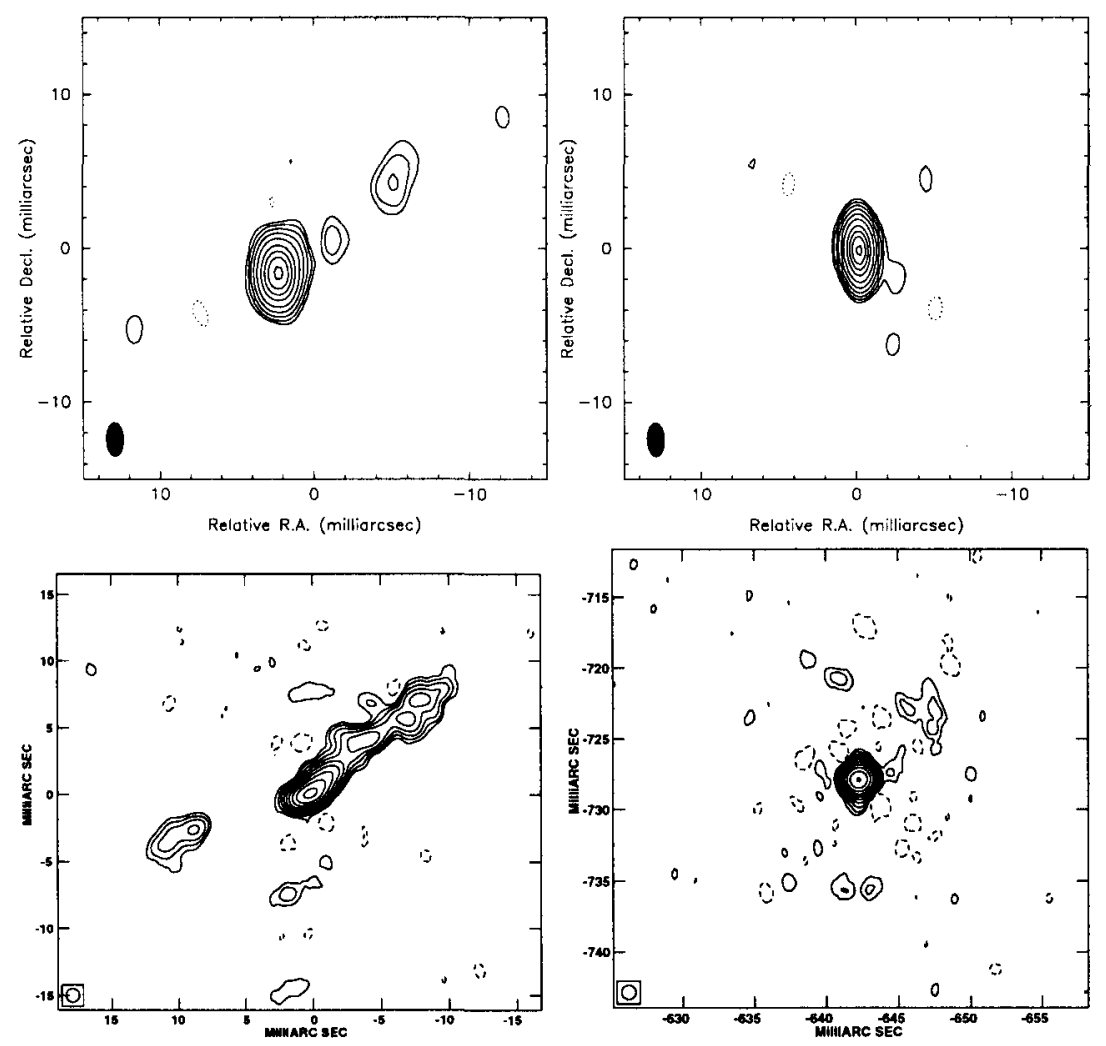

Figure 2. NE (left) and SW (right) images of 1830-211. Top: VLBA at $22 \mathrm{GHz}$ (from Jones et al. 1996); Bottom: VLBA at $15 \mathrm{GHz}$ (from Garrett et al. 1997)

and by measuring the time delay between variations of the background object as seen in the images (Refsdal 1964). Garrett et al. (1994a) have determined the relative magnification matrix for $0957+561$ (which describes the transformation of an element of one image into another) and also the matrix spatial derivatives along the jet direction. These have been used by Grogin \& Narayan (1996) to model the lens of $0957+561$. Campbell et al. (1995) present the results from several $5 \mathrm{GHz}$ global VLBI observations of $0957+561$. Hybrid maps of the A and B image jets, from 1992 observations, are shown in Figure 4. Their VLBI monitoring of the inner core-jet structures has been used to make an independent estimate of the time delay between variations, of $\sim 1$ year. This is close to the value derived from both optical and radio VLA studies (e.g., Haarsma et al. 1997 ), and taken with the model gives a Hubble constant of $\sim 85 \mathrm{~km} / \mathrm{s} / \mathrm{Mpc}$. 


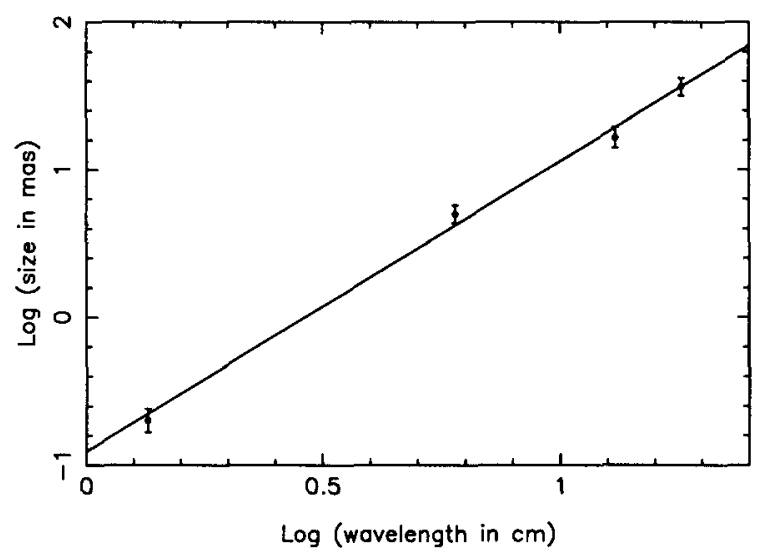

Figure 3. Variation of $1830-211 \mathrm{SW}$ image size with wavelength (from Jones et al. 1996)

The small image separation ( 335 mas) lens system $0218+357$ also shows promise as a tool for determining the Hubble constant, since short timescale variations in percentage polarization have been reported from VLA observations (Corbett et al. 1996), leading to a provisional value for the time delay between the images of 12 days. High resolution studies of this system have been made with the VLBA by Patnaik et al. (1995) and Porcas \& Patnaik (1996a,b). Maps of the $\mathrm{A}$ and $\mathrm{B}$ images at $15 \mathrm{GHz}$ are shown in Figure 5. At high frequencies, each image consists of 2 main components-a western core component, and a steeper spectrum knot. Components in the A image show the characteristic "tangential stretching" in $\mathrm{PA}-40^{\circ}$. The $15 \mathrm{GHz}$ observations have been used to derive the image relative magnification matrix. No motion of the knot with respect to the core was detected over a 1.8 year period. At frequencies of $5 \mathrm{GHz}$ and lower, the structures of both images become larger and more amorphous, whilst image A continues to exhibit extended structure in $\mathrm{PA}-40^{\circ}$; the compact core component becomes undetectable. Nair (1996) has given a model for this system, incorporating the VLBI structure constraints.

The quad lens system MG $0414+0534$ consists of 2 close, bright images, A1 and $A 2$, and weaker images $B$ and $C$. Unfortunately, there seems to be very little variability in the background source, necessary for time delay measurements. Porcas, Patnaik and Garrett have studied the images of this system with the EVN at 1.6 and $5 \mathrm{GHz}$ (see Figure 6, and Patnaik \& Porcas, 1996). All images show a dominant, core-like component at both frequencies. Image $\mathrm{B}$ shows a jet-like extension in PA $130^{\circ}$ and suggestions of a counter-jet at $5 \mathrm{GHz}$. Image $\mathrm{C}$ is barely resolved in the $\mathrm{N}$-S direction at $1.6 \mathrm{GHz}$, but exhibits a knot-like component in $\mathrm{PA}-5^{\circ}$ at $5 \mathrm{GHz}$. Images $\mathrm{A} 1$ and $\mathrm{A} 2$ both show a complex structure, with both tangential and radial extensions, which is poorly imaged in our $5 \mathrm{GHz}$ maps. Recent higher-resolution VLBA maps at $5 \mathrm{GHz}$ (C. Trotter et al. , in preparation) show that the roughly colinear core-jet structure seen in images C and D is dramatically distorted in A1 and A2. Falco et al. (1997) have modeled this system using parameters derived from HST and radio data. 

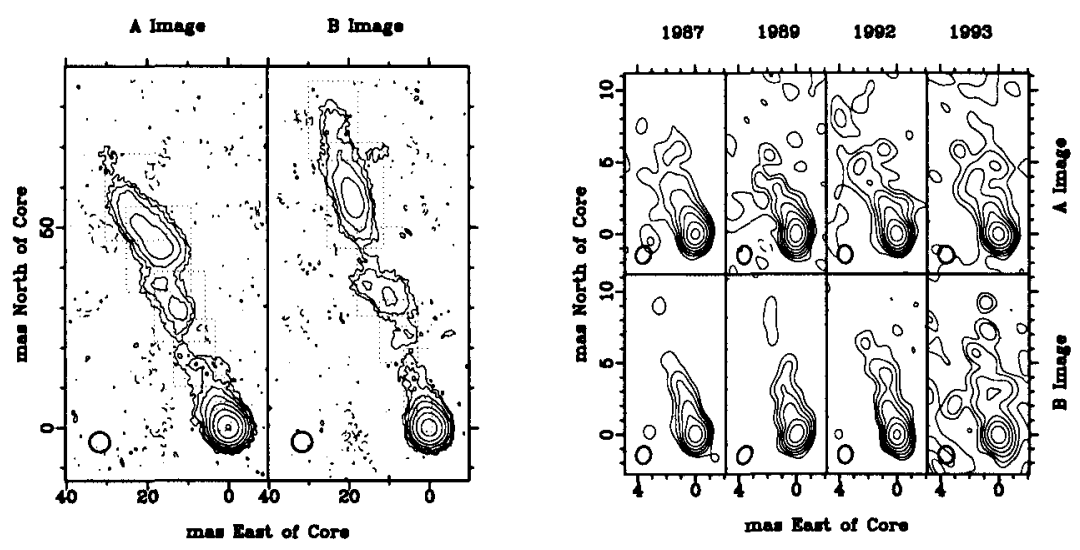

Figure 4. $5 \mathrm{GHz}$ global observations of 0957+561 (from Campbell et al. 1996); (left) Structures of images A and B; (right) Variation of the inner cores of A and B at 4 epochs.

Observations of two other quad systems are presented elsewhere in these Proceedings: $1608+656$ (Myers et al. 1995) by Fassnacht et al. (these Proceedings, p. 311) and $1422+231$ by Patnaik \& Porcas (these Proceedings, p. 319). There are no published VLBI observations of the double $1600+434$ (Jackson et al. 1995).

\section{Polarization, Astrometric, and Spectral Line VLBI}

The added complexity of such observations can be well rewarded in gravitational lens studies. Gravitational lensing preserves the polarization percentage and angle of each region of the object in all images, providing Faraday rotation and depolarization along the different image paths is taken into account. This thus provides a useful label for identifying corresponding regions in different images. Any position offset between the brightness peaks in polarized and unpolarized emission can also be used as a vector to help determine the image relative magnification matrix. And finally, any unexplained misalignment between the polarization angle and jet position angle in single-image objects may indicate structural distortion from an unrecognized lens near the image path (e.g. Hutchison \& Cawthorne, these Proceedings, p. 125). Observations of $0218+357$ at 8.4 and $43 \mathrm{GHz}$ (Kemball, Patnaik, \& Porcas, in preparation) have shown that most of the polarized emission comes from its core component, and confirm that the two image paths have very different Faraday rotations.

Astrometric measurements of the separation between lens images provide an interesting instance where maps at different frequencies or epochs can be reg- 

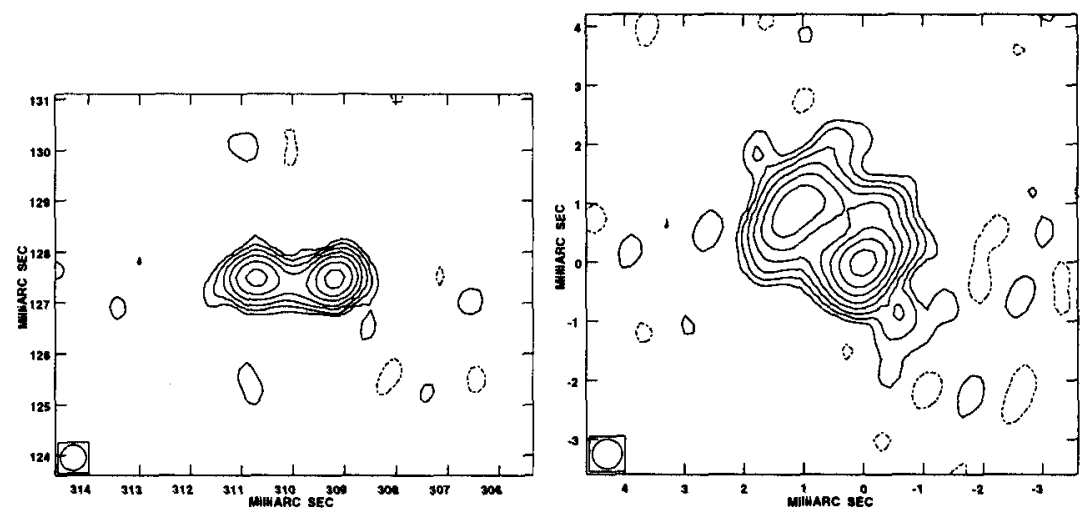

Figure 5. 0218+357 images B (left) and A (right) from VLBA at $15 \mathrm{GHz}$ (from Patnaik et al. 1995)

istered unambiguously (Porcas \& Patnaik 1995). Some of the normal difficulties of such a registration are investigated in Rioja \& Porcas (these Proceedings, p. 95). In general, the vector separation between corresponding points in two images is unique to that point within the images. The achromatic property of the relative magnification matrix thus allows a separation measurement between points at one frequency to locate the points in images at another. The same technique can be used to register maps at different epochs to identify moving and stationary components in sources with structural variations. Kochanek et al. (1996) have pointed out that sufficiently accurate measurements of a stable source can also be used to detect the proper motion of the lens itself, on times-scales of a decade.

Gravitational lens systems provide a special opportunity to study the distribution of absorbing gas across the image paths through the lens, using spectral line VLBI. This topic is discussed by Carilli et al. (these Proceedings, p. 317)

Acknowledgments. I thank Ian Browne, Michael Garrett, Dayton Jones, Josef Lehár, Alok Patnaik, Cathy Trotter, and Peter Wilkinson for help with preparing this review. The National Radio Astronomy Observatory is a facility of the National Science Foundation, operated under a cooperative agreement by Associated Universities, Inc.

\section{References}

Campbell, R. M. et al. 1995. $A J$, 110, 2566-2569.

Corbett, E. A. et al. 1996. in IAU Symp. 173: Astrophysical Applications of Gravitational Lensing. eds.C. Kochanek \& J. Hewitt (Dordrecht: Kluwer), 37-42.

Falco, E. E., Lehár, J., \& Shapiro, I. I. 1997. AJ, 113, 540-549.

Garrett, M. A. et al. 1994a. MNRAS, 270, 457-464.

Garrett, M. A., Patnaik, A. R., \& Porcas, R. W. 1994b. in Proc. 2nd EVN/JIVE Symposium, eds. A.J.Kus, R.T.Schilizzi, K. M. Borkowski \& L. I. Gurvits (Torun: TRAO),73-77.

Garrett, M. A. et al. 1996a. MNRAS, 279, L7-11.

Garrett, M. A. et al. 1996b. in IAU Symp. 173: Astrophysical Applications of Gravitational Lensing. eds.C. Kochanek \& J. Hewitt (Dordrecht: Kluwer), 189-190.

Garrett, M. A. et al. 1997. Vistas in Astronomy, eds. M.A.Garrett \& F. Colomer, 41, 281-285.

Grogin, N. A., \& Narayan, R. 1996. ApJ, 464, 92-113. 

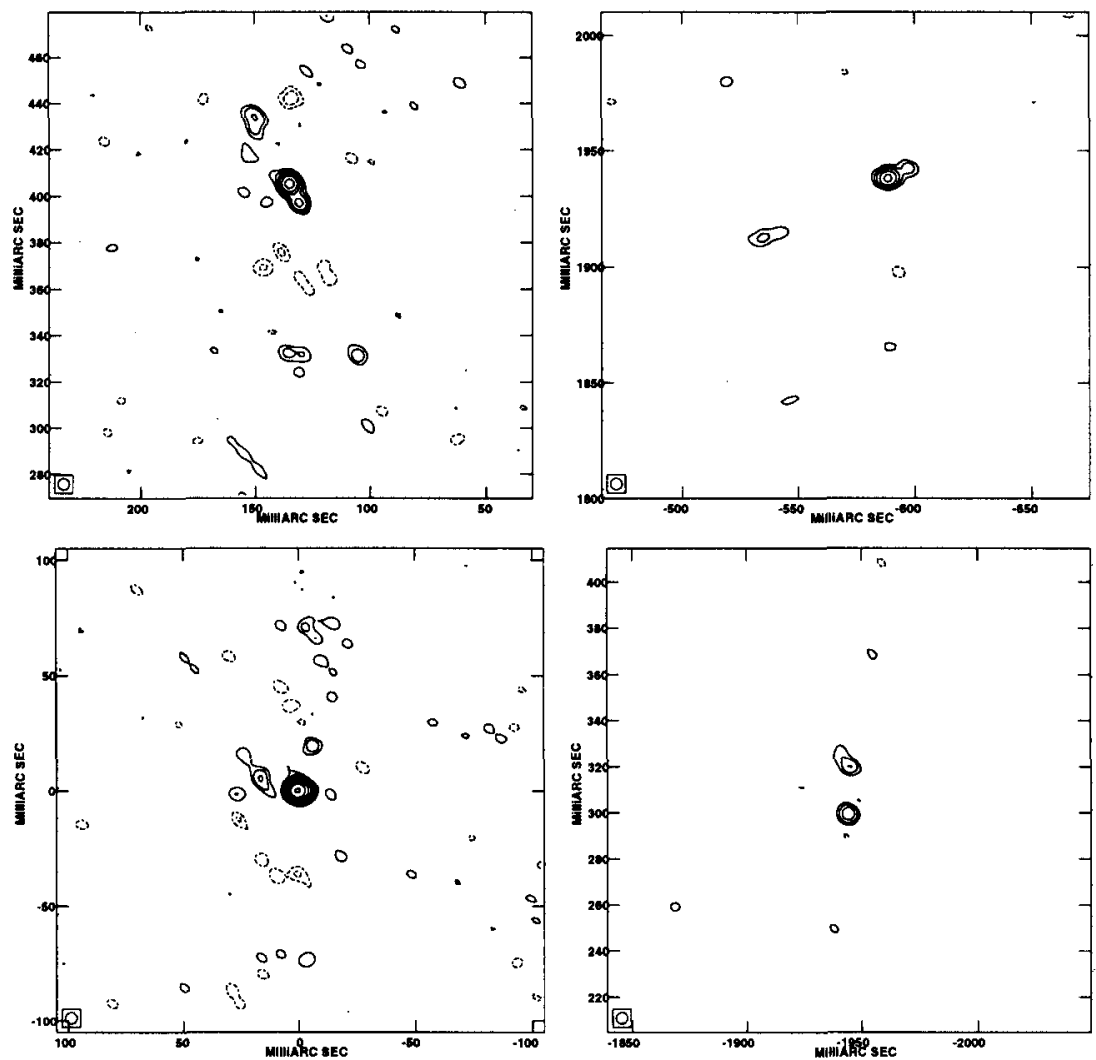

Figure 6. EVN $5 \mathrm{GHz}$ maps of MG 0414+0534; (clockwise from bottom left) images A1, A2, B, C (from Porcas, Patnaik, \& Garrett, in preparation)

Haarsma, D. B. et al. 1997. $A p J, 479,102-118$.

Jackson, N. et al. 1995. MNRAS, 274, L25-29.

Jones, D. L. et al. 1996. $A p J, 470$, L23-25.

King, L. J. et al. 1997. MNRAS, 289, 450-456.

Kochanek, C. S., Kolatt, T. S., \& Bartelmann, M. 1996. ApJ, 473, 610-619.

Lovell, J. E. J. at el. 1996. $A p J, 472$, L5-7.

Myers, S. T. et al. 1995. ApJ, 447, L5-8.

Nair, S. 1996. in IAU Symp. 173: Astrophysical Applications of Gravitational Lensing. eds.C. Kochanek \& J. Hewitt (Dordrecht: Kluwer), 197-198.

Nair, S., \& Garrett, M. A. 1997. MNRAS, 284, 58-72.

Patnaik, A. R., \& Porcas, R. W. 1996. in IAU Symp. 173: Astrophysical Applications of Gravitational Lensing. eds.C. Kochanek \& J. Hewitt (Dordrecht: Kluwer), 305-310.

Patnaik, A. R., Porcas, R. W., \& Browne, I. W. A. 1995. MNRAS, 274, L5-7.

Patnaik, A. R. et al. 1996. in IAU Symp. 173: Astrophysical Applications of Gravitational Lensing. eds.C. Kochanek \& J. Hewitt (Dordrecht: Kluwer), 405-406.

Porcas, R. W. 1994. in Compact Extragalactic Radio Sources, eds. J. A. Zensus \& K. I. Kellermann (Green Bank: NRAO), 125-128.

Porcas, R. W. et al. 1979. Nature, 282, 385-386. 
Porcas, R. W., \& Patnaik, A. R. 1995. in Proc. 10th Working Meeting on European VLBI for Geodesy \& Astrometry, eds. R.Lanotte \& G. Bianco (Matera:ASI), 188-192.

Porcas, R. W., \& Patnaik, A. R. 1996a. in IAU Symp. 173: Astrophysical Applications of Gravitational Lensing. eds.C. Kochanek \& J. Hewitt (Dordrecht: Kluwer), 311-316.

Porcas, R. W., \& Patnaik, A. R. 1996b. in IAU Symp. 175: Extragalactic Radio Sources, eds. R. Ekers, C. Fanti, \& L. Padrielli (Dordrecht: Kluwer), 115-117.

Refsdal, S. 1964. MNRAS, 128, 307-310.

Wambsganss, J., \& Paczynski, B. 1992. ApJ, 397, L1-4.

Wilkinson, P. N. et al. 1996. in IAU Symp. 168: Examining the Big Bang and Diffuse Background Radiations, eds. M. Kafatos \& Y. Kondo (Dordrecht: Kluwer), 95-98. 